\title{
Entre flujos y fronteras: la educación superior mediada tecnológicamente vista a través de una perspectiva etnográfica'
}

\author{
Julio Ernesto Rojas Mesa ${ }^{2}$ \\ Linda Alejandra Leal Urueña ${ }^{3}$
}

\begin{abstract}
Resumen
El artículo aborda, desde una perspectiva etnográfica, el análisis de tres categorías utilizadas en la historia de la educación superior contemporánea: educación desescolarizada, educación a distancia y educación virtual. Este proceso se entiende como una formación discursiva que consolida el discurso de lo que actualmente definimos como educación virtual en la educación superior colombiana.
\end{abstract}

Palabras clave: educación superior, etnografía, educación desescolarizada, educación a distancia, educación virtual, prácticas pedagógicas.

\footnotetext{
1. Este artículo hace parte del trabajo de tesis doctoral titulado: Educación virtual: del discurso a las prácticas pedagógicas. Estudio etnográfico en tres instituciones de educación superior en Colombia, elaborado como requisito de grado para el programa «Teoría de la Educación y Pedagogía social» de la UNED-España. Dicho estudio pretende adelantar un análisis de la relación entre educación superior y TIC, enfocándose para ello en el estudio etnográfico de tres universidades colombianas: la universidad Pedagógica Nacional, la Universidad Nacional Abierta y a Distancia y la Universidad Santo Tomas. Adicionalmente, ha sido complementado con el desarrollo del proyecto «Taller de gestión de conocimiento local a partir de relaciones entre Educación y Tecnología», adelantado por la Corporación Bohíos.

2. Ph.D. Teoría de la Educación. Docente investigador. Universidad Nacional Abierta y a Distancia - UNAD (Colombia). Correo: julio.rojas@unad.edu.co

3. M.Sc Tecnologías de Información aplicadas a la Educación. Docente Auxiliar. Universidad Pedagógica Nacional (Colombia).
} 


\title{
Between flow and borders: higher education mediated by technology looked through a ethographic perspective
}

\begin{abstract}
This paper discusses, from an ethnographic perspective, the analysis of three categories used in the history of the higher education in the present time: deschooled education, distance education and virtual education. This process is understood as a discursive formation that consolidates the discourse of what we now define as virtual education in the Colombian higher education.
\end{abstract}

Keywords: higher education, ethnography, deschooled education, distance education, virtual education, pedagogical practices.

Recibido: 13/11/2014

Aceptado: 25/11/2014

De todo, quedaron tres cosas: la certeza de que estaba siempre comenzando, la certeza de que había que seguir, y la certeza de que sería interrumpido antes de terminar. Hacer de la interrupción un camino nuevo, hacer de la caída un paso de danza, del miedo una escalera, del sueño, un puente, de la búsqueda... un encuentro.

(F. Pessoa)

\section{lntroducción}

Los procesos de virtualización de la educación superior han sido ampliamente estudiados desde enfoques pedagógicos y de investigación cualitativa. La etnografía ha sido uno de los tantos enfoques metodológicos a partir de los cuales se han realizado análisis sobre este devenir de la relación TIC-EducaciónCultura.

El objetivo de este trabajo consiste en ir un poco más allá de la comprensión etnográfica como un levantamiento y descripción de datos en «terreno o campo» tradicional y abordar el enfoque de la etnografía aplicada en ambientes digitales. Se aborda la tarea de describir la transición de la noción cartográfica y física del campo trabajada desde la etnografía clásica, hacia una noción de campo virtual y telecomunicativa. 
Para analizar cómo se fue consolidando un discurso sobre la educación virtual en la política educativa y la universidad colombiana, se describen tres formaciones discursivas construidas en la historia de la educación superior contemporánea: 1. Desescolarización y educación abierta y a distancia, 2. Semipresencialidad y 3. Educación virtual.

En la primera parte del texto se realiza una descripción del contexto investigativo etnográfico en torno a la relación tecnología y cultura. La segunda parte desarrolla el análisis etnográfico de la transición entre la categoría de desescolarización, pasando por la de semipresencialidad hasta llegar a la de educación virtual.

\section{Revisión de experiencias investigativas en la relación cultura y tecnología en la investigación etnográfica}

Es de amplio consenso que la etnología /etnografía, está caracterizada como un campo de carácter fronterizo, situado el borde de las representaciones epistemológicas de las múltiples disciplinas científicas (Restrepo, 2008). Este hecho teórico y metodológico de la etnografía fue consolidado en el pasado siglo, a partir de lo que se conoció como la triple crisis de la Antropología: crisis de método, de objeto y de representación (Velasco \& Díaz de Rada, 1997; Restrepo, 2006; Flórez, 2007).

Comenzó a ser claro que «el otro» no era un paradigma simbólico y exótico encarnado en un ser humano con características sociales, políticas, económicas, étnicas y culturales diferentes, ubicado en algún remoto lugar, sino que por el contrario, el «otro» era básicamente una construcción simbólica del sujeto existente en las sociedades propias, en su sentido fronterizo y en su multiplicidad cultural (Auge, 1995; Mayans i Planells, 2002; Turkle, 2007).

Esta reflexión estuvo asociada con un siglo XX lleno de procesos de migración, producto de siglos de colonización, de reorganización de identidades individuales y colectivas y de construcción social y cultural de ambientes de multiculturalidad e interculturalidad en todas las sociedades modernas.

La revolución telecomunicativa y los ambientes digitales contribuyeron a llamar la atención sobre las profundas transformaciones en la percepción territorial, política, social, cultural, económica y filosófica que ocurría desde fines del siglo XX (Castells, 2002; 2010; Bauman, 2007).

Esta nueva era propuso la construcción de representaciones simbólicas ancladas a la percepción, uso e interacción en ambientes digitales, revolucionó la noción de tiempo y espacio, de territorialidad y disolvió de cierta manera el concepto de límites en nuevos paradigmas de reticularidad y ubicuidad emergidos con estas tecnologías (Bauman, 2005; 2011). 
Entre flujos y fronteras: la educación superior mediada tecnológicamente vista a través de una perspectiva etnográfica . Artículo producto de la investigación.

Sin embargo, lo que hace tan vistoso y a su vez tan pavoroso este descentramiento de la condición corporal humana en la época contemporánea, tiene que ver con la manera como la sociedad afronta esos eventos de borde. Al respecto se ha generado un intenso y variado debate.

Metodológicamente, las retrospectivas realizadas por Díaz Cruz en torno a los estudios antropológicos que describen la relación tecnología, artefactos y cultura plantea la complementariedad entre el modelo de los sistemas socio técnicos y los métodos de análisis procesalistas trabajados en la antropología política (1995), el estado de arte realizado por Escobar sobre Ciber-antropología (2005), da cuenta de los estudios realizados en torno de la relación entre antropología y tecnologías, especialmente sobre las tecnologías electrónicas.

De otro lado, los estudios historiográficos han aportado abundante material para el análisis etnográfico de la relación cultura y tecnología (Ronderos y Valderrama 2003, Hirsh 2011). Se resalta el trabajo de análisis histórico de Staudenmaier (2002), sobre el determinismo tecnológico y sus impactos en la sociedad. Este investigador se empeñó en construir una genealogía de la historia de la tecnocultura en los últimos cuarenta años (Moon, 2011).

Los trabajos de Turkle son significativos. Esta investigadora se dedicó a analizar la emergencia de múltiples identidades tecno culturales como resultado del uso de comunicaciones mediadas por ordenador (Turkle \& Papert, 1991; Turkle, Taggart, \& Kidd, 2006; Turkle, 2007; 2009; 2011).

Así mismo, surgió el esfuerzo por elaborar ejercicios teóricos y epistemológicos de la disciplina llevados al lugar de la teorización conceptual y metodológica de una etnografía aplicada en ambientes digitales. Hine (2004; 2005), desarrolla su trabajo en torno a la investigación en los ambientes digitales, define la etnografía virtual como un campo de conocimiento que utiliza métodos y técnicas de la etnografía clásica pero también se asienta en construcciones teóricas y metodológicas propias de su incursión en los ambientes digitales. Describe el fenómeno de la E-Ciencia como un escenario emergente en el que trabaja no solamente la etnografía sino todas las disciplinas científicas de las ciencias sociales y exactas $(2007 ; 2008)$. Su trabajo es secundado por el grupo de entovirtual en España, y su preocupación por encontrar la categoría teórica que defina una etnografía que se desarrolla en los ambientes digitales (Domínguez, Estaella, \& Gómez, 2007).

Los trabajos de Rueda son importantes en la descripción de una perspectiva latinoamericana de la cibercultura, la ciudadanía digital y el empoderamiento de las comunidades a través de las TIC (Rueda, 2008, Rozo, 2008b, Rojas y Bernal, 2008). 
Mayans creó el observatorio para la Cibersociedad (OCS) a partir de sus primeras reflexiones sobre el ciberespacio publicadas Genero Chat (2002). Este espacio dio pie para múltiples reflexiones en torno al fenómeno Ciber como un triángulo de discursos, prácticas y ambientes en la cultura y la sociedad. Esta nueva generación de etnógrafos cuyo «campo» son los ambientes virtuales y digitales, terminó creando un abanico de categorías organizadas bajo tres nodos principales: Investigación, Desarrollo y Aplicaciones (Observatorio para la CiberSociedad (OCS) 2013).

Rojas, desarrolla una serie de análisis en la transformación del sujeto, su entorno sociocultural y sus procesos de producción de saber. Para Rojas, el uso de artefactos electrónicos ha perfilado un sujeto rizomático, ubicuo y prosumidor (Rojas Mesa \& Salazar Manrique, 2012). En otros trabajos, Rojas identifica las repercusiones de la política de TIC en el sector educativo y la manera como se construye una nueva cultura académica soportada en los discursos, prácticas y territorialidades mediadas por TIC (Rojas, 2013).

Downes (2008), describió cambios estructurales en campos de relación como la tecnología y la educación. Este autor propone una tensión producida entre dos tendencias: Contenido Vs Interacción, y abre una gruesa línea de transformación de la cultura tecnológica en la educación a través de los MOOC (Downes, 2012). Los trabajos de Maldonado y Leal acerca de los grados de interacción en foros y la construcción y fortalecimiento de comunidades de aprendizaje, han permitido definir el sentido que toman las interacciones dentro de los ambientes digitales en un proceso académico (Maldonado et al., 2006, Maldonado Granados y Leal Urueña, 2009).

Indudablemente, el enfoque de trabajo de campo, instaurado por la etnografía clásica, se ha transformado enormemente. La sociedad científica contemporánea asiste al surgimiento de una noción vectorial telecomunicativa de la concepción del campo en la etnografía.

Todo ello, permite analizar las instituciones educativas, entender cómo construyen su lugar en estas nuevas narrativas de la investigación social sobre los ambientes digitales. 


\section{La entrada de la desescolarización en las universidades estudiadas}

La historia contada en este trabajo no es lineal y esta dimensión de lo heterogéneo dentro de un discurso homogéneo y formal, que para el caso de la educación consistía en un sistema organizado presencial y secuencialmente sobre el eje de enseñanza-aprendizaje, centrado en la figura catedrática del docente, aunque tuvo experiencias desde comienzos del siglo XX, tomo fuerza y empezó a deslocalizarse en Colombia desde mediados de los años 70 con la realización de los primeros cursos universitarios en programas de educación desescolarizada, abierta y a distancia (EAD) (Rojas Mesa, 2003).

La definición de lo extraescolar en la educación superior colombiana estuvo precedido por las prácticas de Educación de Adultos desde 1942 con las escuelas radiofónicas de Sutatenza ${ }^{4}$ y que se articulaban en un eje local/global del discurso sobre la relación entre sujetos y educación planteada por la UNESCO en 1949 (UNESCO, 1949) y materializadas en enunciados jurídicos institucionales nacionales muchos años más tarde (González, 2006; Sarmiento, 2007; Angel, 2008).

El surgimiento del modelo EAD se dio a causa de varios factores: cambios sociopolíticos, altos costos de la educación convencional, consolidación de una noción de life-long-learning, el avance de las teorías educativas y las tecnologías telecomunicativas.

Todo ello, en su conjunto, registró el cambio del conjunto de discursos/prácticas y ambientes de las sociedades que impactaban escenarios políticos, económicos, sociales, culturales, territoriales, tecnológicos y educativos proyectando un debate teórico en la prospectiva educativa que a la fecha es intenso (García Aretio, 2011).

Para las universidades de este estudio, el campo se configuró a partir de una cartografía geográfica que se fue disolviendo en el surgimiento de una cartografía virtual como se denomina teóricamente a la noción de campo en este trabajo.

Según acta de octubre 13 de 1975, se creó en la Universidad Santo Tomas (de ahora en adelante USTA), el Programa académico de Enseñanza Desescolarizada, a través del Consejo de provincia de San Luis Beltrán de Colombia, «como una respuesta a las necesidades más apremiantes del entorno social, regional y como innovación de aplicaciones pedagógicas contemporáneas (...) (...). Así comenzó a funcionar en Colombia el primer programa de Educación Superior a Distancia totalmente desescolarizado» (Pita, 2006: 94). En 1976, esta universidad comienza la estructuración del Centro de Enseñanza Desescolarizada - CED-, y abre los primeros Centros Regionales en Bucaramanga, Ibagué, Cartagena, Cali y Medellín. 
Muchos de los desarrollos curriculares pensados y diseñados para los programas desescolarizados, así como la idea de su modelo educativo, terminaron alimentando gran parte del desarrollo curricular de los programas presenciales de la universidad (USTA, Vicerrectoría Académica, 2009: 14).

Se aceptaba que la USTA a través de un complejo sistema de medicaciones y una incipiente tecnología educativa, podía romper con la idea de claustro que había acompañado a su oferta educativa durante casi 500 años en Bogotá «la tomística del nuevo reino de granada, definida como Universidad de Estudio General por la Bula Fundacional de 1580» (USTA, 2004: 42).

En el caso de la Universidad Pedagógica Nacional (UPN) se asumió un silencio institucional en relación con estos discursos de desescolarización. Por el contrario, la Universidad se dedicó más bien a fortalecer la práctica educativa dentro de la modalidad presencial no sin advertir sobre el riesgo de permitir la imposición de la corriente de «Tecnología educativa» (UPN, 2006; Martínez Boom, 2004). Esta posición tomada en la UPN, estuvo apoyada por una opinión académica visiblemente opuesta al tema de la EAD (Lopera, 2002).

La incursión de la tecnología educativa se concibió como una reforma en contra del quehacer docente: «los técnicos del ministerio de educación quienes obsesionados por el cumplimiento de "objetivos instruccionales", negaban la voz y el pensamiento a los maestros, reducían el proceso de aprendizaje al cumplimiento de objetivos "observables" predeterminados por la Tecnología educativa y el diseño instruccional (TEYDI)» (Tamayo, 2006: 102).

Para el momento en la UPN, entidad encargada de orientar «la investigación, el desarrollo educativo y la formación docente de conformidad con las necesidades prioridades nacionales» (Presidencia de la República, 1980, Art. 136), se reconocían básicamente tres grandes sistemas de educación: formal, no formal e informal y los modelos desescolarizados no se incluían en la educación formal. En la UPN se comenzó un proceso de construcción de la epistemología de la pedagogía y el empoderamiento del docente como sujeto de saber/poder (Martínez Boom, 2004). Ello implicó que el docente establecía de manera exclusiva la mediación, bien sea con el uso de medios tecnológicos o bien a través de la cátedra magistral dentro de un paradigma del «maestro de tiza y tablero» (Tamayo, 2006: 106).

En la tercera perspectiva de este estudio: la UNAD, hablar de desescolarización significa hablar de Educación a Distancia (Rojas Mesa, 2003).

El alto porcentaje de población excluida del sistema educativo formal presencial, explica el surgimiento de la UNAD, antigua UNISUR. Su creación fue el resultado de una presión generada por comunidades del sur de la ciudad de Bogotá, organizadas en Juntas de Acción Comunal, con apoyo de representantes políticos. 
Entre flujos y fronteras: la educación superior mediada tecnológicamente vista a través de una perspectiva etnográfica . Artículo producto de la investigación.

La UNAD es la metáfora más específica y precisa de la combinación de distancia y presencia en la práctica educativa; la universidad del «sur» de Bogotás. Establecimiento público de orden nacional, con domicilio en la ciudad de Bogotá, y con sede en el sur de la ciudad.

Teóricamente, UNISUR era inicialmente considerada un filtro para resolver un problema de marginalidad social que fue entendida literalmente como una población extramuros del sistema educativo formal.

El Consejo Superior de esta institución estaba conformado por los miembros establecidos estatuariamente por el gobierno para las instituciones universitarias y adicionalmente un representante de las Juntas de Acción comunal del sur de Bogotá; para su elección se demarcó lo que se debía considerar el territorio Sur de la ciudad a través del decreto 1782 de 1987: «Los presidentes de las juntas de Acción Comunal, tendrán derecho a elegir el representante de la comunidad de los barrios del sur para el Consejo Superior de la Unidad Universitaria del Sur de Bogotá, los cuales corresponden a los barrios situados al Sur de la calle primera de la nomenclatura urbana del Distrito Especial de Bogotá» (Ministerio de Gobierno, 1987). El representante de las Juntas de Acción Comunal podía perder su calidad de representante, entre otras causas, por: «cambio en la residencia de los barrios al sur de la nomenclatura urbana del Distrito Especial de Bogotá» (Ministerio de Gobierno, 1987, art. 6, Num. 2).

Finalmente, el enunciado jurídico-administrativo de la política educativa a distancia estatal hace presencia en tres momentos. Inicialmente, crea la Unidad Universitaria del Sur de Bogotá UNISUR, a través de la ley 52 de 1981 (Congreso de la República de Colombia, 1981), un año más tarde, formaliza el concepto de educación Abierta y a Distancia e inserta el de desescolarización al interior de los planes de estudio con el decreto 2412 de 1982 (Presidencia de la República de Colombia, 1982, Art. 1) y finalmente crea el Sistema de Educación Superior a Distancia (SEAD) a través del decreto 1820 de 1983 (Presidencia de la República, 1983), convocando a universidades públicas y privadas a participar en él.

Mientras UNISUR (futura UNAD) y la USTA, participaban de este sistema, la UPN tomó distancia reafirmando de manera contundente el modelo presencial de educación escolarizada.

Hasta aquí la noción de campo que se delinea en la investigación está caracterizada de un lado, por una universidad privada Bimodal (USTA) cuya proyección regional desescolarizada se materializó en la figura de Centros Regionales ubicados en ciudades intermedias del país, de otro lado, una universidad pública nacional (UPN) con una sola sede ubicada exclusivamente en Bogotá, la capital de la república y que desarrolla su oferta educativa en modalidad presencial, la

5. Indudablemente, en la geografía urbana bogotana, el sur de la ciudad representa el sector de la ciudad con menos recursos económicos, reducidas o nulas posibilidades de acceso a servicios públicos (Salud, agua potable, saneamiento básico, vivienda, educación). 
cual fue el centro para proponer la política educativa nacional en consonancia con el encargo jurídico realizado por su estatuto de creación. Finalmente, se encuentra la UNAD que materializa el objetivo del Estado de experimentar con la modalidad educativa a distancia para resolver el problema de acceso al sistema educativo de un alto porcentaje de población de escasos recursos.

\section{Distancia y presencia en la noción de campo: la semipresencialidad en la retórica educativa colombiana}

Con la formalización jurídica del eje presencia/distancia en los programas de educación superior colombiana, en 1982 apareció en el discurso jurídico educativo del Estado la posibilidad de una doble modalidad en la oferta educativa de las universidades (presencial y a distancia).

Sin embargo, hablar de distancia y presencia en relación con las mediaciones tele-comunicativas de los años 80 (correo certificado, teléfono, radio, televisión), planteó el reto de desplazamiento del campo espacial a un campo telecomunicativo, del cual sus protagonistas no tenían aún plena consciencia, hecho que generó una interface adicional: la semipresencialidad.

La dimensión del campo universitario estudiado, aunque tenía una base fuertemente cartográfica -en la década del 80 la USTA y UNISUR comenzaron a abrir Centros regionales de Educación a Distancia en varios sitios del país- estaba soportada ahora especialmente por una acción tele comunicativa sincrónica permanente (el teléfono).

El territorio físico y simbólico de estas universidades se había expandido y tenía una noción de continuidad real, instaurada en una dimensión tecnológica sincrónica. Pero la realidad de dicha dimensión territorial no era ni exactamente cartográfica ni la dimensión comunicativa era necesariamente física (cara/cara). Surgió un archipiélago de sedes físicas, conectadas tele comunicativamente (UNESCO, 1989; 1996).

Esta tensión de la presencia universitaria en la distancia generada por el intervalo geográfico de sus centros regionales de atención, dio pie a posteriores debates acerca de lo que significaba la presencia o la distancia y su impacto en el desarrollo educativo, al punto que la ley, dictaminaba que a pesar de que los programas a distancia se caracterizaban por la alta autonomía del estudiante en su proceso de formación y el apoyo de los medios tecnológicos, era imperativo que la universidad se comprometiera con un acompañamiento tutorial presencial periódico a los estudiantes (Presidencia de la República de Colombia, 1982; 1983; Congreso de Colombia, 1992). 
Desde la institucionalidad, la UNAD denunció este hecho jurídico como la interdicción al modelo educativo a distancia. Criticó el monopolio de representaciones sobre lo educativo relacionadas con un ideal universal educativo centrado en la enseñanza y la presencia. Es significativo el análisis del profesor Roberto Salazar al respecto: «Son conocidas las denominaciones que se le han venido dado al agrupamiento de las horas de acompañamiento tutorial en educación a distancia: educación basada en encuentros intensivos, compactos, concentrados, o educación a distancia con apoyo semipresencial» (Rojas Mesa, 2013).

Etnográficamente el fenómeno es muy interesante. El enunciado jurídico instaura a partir de los años 80 el concepto de distancia en el mundo educativo universitario. Sin embargo los discursos educativos imperantes son la presencia, la enseñanza y el docente ¿Cómo resolver esta discontinuidad?

La EAD, que en 1982 se definió como «conjunto de actividades (...) de acuerdo con planes de formación o capacitación, total o parcialmente desescolarizados» (Presidencia de la República de Colombia, 1982), en 1983 se convierte en el sistema de EAD con enganche presencial. Es decir, no eran programas totalmente a distancia ni tampoco el modelo era exclusivamente centrado en el aprendizaje. En primer lugar se crean los centros regionales, zonales o locales de atención a estudiantes «Dichos centros no tendrán el carácter de seccionales» (Presidencia de la República, 1983, Art.7).

En segundo lugar se otorga al estudiante el estatus de aprendiz, es decir surge formalmente el concepto de aprendizaje «La Educación Superior Abierta y a Distancia fomenta la capacidad de la persona para aprender por sí misma los contenidos de la Educación Superior, siempre que tenga las suficientes aptitudes intelectuales y los hábitos personales de estudio, constancia y autodisciplina» (Presidencia de la República, 1983, Art.3 paragr.2).

La cartografía del proceso queda clarificada de la siguiente manera: el centro creado por la universidad tiene una característica de atención académica/ administrativa y el estudiante territorializa su cuerpo como la infraestructura para un proceso de aprendizaje. En este sentido la universidad hace presencia con cada estudiante que formaliza su proceso educativo con ella. El modelo universal es la enseñanza, aunque el estudiante se empodera en el aprendizaje el docente es la figura central del proceso educativo y se conecta con el estudiante en escenarios presenciales denominados acompañamiento tutorial. Este fenómeno explica la manera como se subsana la discontinuidad surgida entre una idea de presencia que representa el ideario educativo universal y la idea de distancia que se entiende como un salto hacia el cuerpo del estudiante/aprendiz como gestor de su propio aprendizaje: 
«En la Educación Superior Abierta y a Distancia, la relación profesor/alumno habitualmente no es presencial sino mediatizada, a través del uso de uno o varios medios de comunicación. No obstante, deberán programarse sesiones presenciales. Por adaptarse con mayor flexibilidad a las circunstancias de lugar y tiempo del alumno, hace posible un acceso más amplio de las personas a los procesos educativos» (Presidencia de la República, 1983, Art.2.).

Con el cambio de nombre de UNISUR por el de UNAD, se formaliza la deslocalización de UNISUR en el sur de Bogotá y le da cobertura nacional (Congreso de Colombia, 1997, Art.1), situación que ya venía ocurriendo pues UNISUR desde mediados de los años 80 , había creado centros regionales a lo largo y ancho del país y había desplegado el modelo de semipresencialidad en el acompañamiento docente a los estudiantes.

¿Cuál es la frontera entre la distancia y la presencia en el proceso educativo?, la semipresencialidad aparece entonces para paliar ese nefasto lugar fronterizo. El concepto de semipresencialidad es reforzado por el decreto 3011 de diciembre de 1997, por el cual se establecen normas para el ofrecimiento de la educación de adultos y se dictan otras disposiciones (Presidencia de la República de Colombia, 1997, Art. 24).

El artículo 24 del decreto 3011, involucra el concepto de semipresencialidad y lo enuncia jurídicamente como un concepto que representa una modalidad educativa independiente de la modalidad abierta y a distancia: «La educación media académica de adultos podrá ofrecerse de manera presencial, semipresencial o abierta y a distancia ${ }^{6} »$. Es absolutamente claro que el enunciado habla de tres metodologías educativas diferenciadas.

Esta disposición permitió legalizar la práctica educativa semipresencial desarrollada en los Centros Regionales de las universidades que ofertaban programas en educación a distancia y presenciales. En otras palabras, la práctica de dinámicas semipresenciales en la oferta educativa desarrollada en las seccionales y Centros Regionales, terminó configurando el ambiente educativo propicio para combinar aspectos específicos de dos modalidades consideradas mutuamente como antagónicas; presencial y a distancia.

Según Rama, para el 2005, el 89\% de las universidades latinoamericanas que ofertaban estudios a distancia y virtuales, presentaban modelos que combinaban elementos presenciales y no presenciales, materiales educativos de diversas generaciones de la EAD, en donde por supuesto también estaban incluidos muchos de los materiales de la metodología presencial (2006). 


\section{Campo físico/campó digital: la virtualización de la educación superior}

Los ambientes digitales entraron inicialmente en la representación de expertos y organismos internacionales como una continuación de la educación a distancia a pesar de que, desde los años 70, existían experiencias consolidadas en el ramo de la informática educativa entendida como una educación presencial impartida con un alto apoyo de tecnologías informáticas (Maldonado \& Maldonado, 2000; Angel, 2004).

A mediados del año 2000, la UNAD establece una ruptura con su tradicional modelo de EAD para incursionar en una nueva representación de la EAD con implementación de TIC y especialmente de ambientes digitales, «El macroproblema formulado indica que la UNAD (antigua UNISUR), no ha desarrollado plenamente su identidad como Universidad Abierta y a Distancia» (UNAD, 2004).

También se describe a la «semipresencialidad» como una práctica docente nociva para la afirmación de una identidad en EAD, continua el profesor Salazar expresando este debate:

«Al mismo tiempo se han generado otros elementos más ideológicos como las teorías de la alienación. El medio aliena, y como el medio aliena la única perspectiva de salvación es la enseñanza directa, que es liberadora. Todo un imaginario ideologizado de la relación directa, en donde la palabra del maestro es la palabra, la mirada del maestro. Lo que yo llamaría en un sentido la pedagogía de los hedores y los humores. Si te coloco en mi axila, entonces, esa colocación opera en términos mecánicos como ese proceso humanizado de formación. Esos procesos puede observarlos uno todavía en la UNAD, a pesar de que es una institución de educación a distancia, en muchos de sus docentes y tutores» (Rojas Mesa, 2013: 294).

Para finales principios de la década del 2000, Ángel definía la educación virtual como un proceso formativo caracterizado por una alta mediación tecnológica y que se producía en ambientes presenciales y a distancia (Angel, 2004). Sin embargo, al final de la década del 2000, define el desarrollo de la EAD en Colombia como un proceso de «Educación semipresencial realizada en sitios remotos», arraigado en el imaginario colectivo y obstáculo para mayores desarrollos de la educación basada en entornos digitales (2008).

El surgimiento del ambiente digital, comenzó a evidenciar la percepción del proceso educativo como un escenario ubicuo y altamente tecnológico:

La «Universidad Virtual» es un proyecto con un nuevo paradigma de enseñanza-aprendizaje, campus y aulas virtuales, autoaprendizaje, bibliotecas electrónicas o videoconferencia con el profesor son algunas de las características que definen la forma de trabajar de docentes y alumnos de 
este nuevo siglo. No tienen aulas ni sillas ni alumnos que asistan a clase. Se trata de espacios cibernáuticos en los cuales los alumnos que se matriculan reciben una cuenta de correo electrónico y la documentación para conocer el funcionamiento y los distintos espacios virtuales de la universidad. Esta Universidad es la que desarrolla y ofrece todos sus servicios a través de Internet, es decir, el alumnado se puede matricular a distancia, y mediante teleformación puede cursar sus estudios y estar en contacto con profesores y compañeros, utilizando una metodología basada en el autoestudio por parte del alumnado, apoyado por tutores a los que pueden dirigir dudas o consultas, normalmente a través de correo electrónico (Pinilla, 2000:3 y 5).

Fue evidente que la modalidad tradicional de EAD comenzó a pasar a revisión. Si bien esta metodología utilizaba una buena parte de mediación tecnológica, se había anclado en una representación dialéctica que la había sumergido en las tormentosas aguas del vacío representado en la distancia, como un antivalor enfrentado a los positivos valores de la presencialidad entendida incluso en el ambiente digital.

En dicho sentido surgieron debates ontológicos acerca del sentido mismo de la categoría de distancia en un proceso educativo. Esta opinión de Luis Facundo Maldonado, director del grupo Tecnice de la universidad pedagógica, recoge el sentido de dicha discusión: "A mi individualmente, el fenómeno de educación a distancia no me puede ir, o sea no me cautiva. Uno está con artefactos acercándose, uno no se está alejando, entonces para que pone como prevalente el concepto de la distancia si en realidad es el encuentro lo que está sucediendo» (Rojas Mesa, 2013: 213) .

Hacia el final de la década del 2000, la UNAD traslada el discurso del aprendizaje hacia modelos de educación virtual y e- Learning. La UPN crea el Instituto de Tecnologías Abiertas en Educación -ITAE, con la intención de comenzar el proceso de reflexión y experimentación de lo que significa la educación virtual y los modelos de e-learning. Finalmente, la universidad Santo Tomas, entra en un proceso de diferenciación entre las modalidades de educación presencial y a distancia quedando atrapada en el proceso de resistencia a la mediación tecnológica como actor protagónico en cualquiera de las dos modalidades.

Se puede decir que la historia de los ambientes es la historia de las fronteras, de los finales y de los comienzos territoriales. El origen no necesariamente es una expresión metafísica histórica, evolutiva que explica ordenadamente la evolución de un proceso o una entidad. La frontera es el lugar del «otro», de la exposición de la alteridad en la que se revitaliza la cultura, se intercambian sentidos y se abortan tradiciones producto del choque de construcciones simbólicas. Más que de lugares, la frontera propone una lectura de flujos, trayectorias, vínculos y relaciones en conflicto y negociación. 
Entre flujos y fronteras: la educación superior mediada tecnológicamente vista a través de una perspectiva etnográfica . Artículo producto de la investigación.

Los debates locales entre metodologías, presencial y a distancia, han permitido continuar con la discusión ontológica. Los primeros estudios se concentraron en buscar la esencia de la educación virtual, por ello la pregunta de investigación del momento era ¿Qué es la educación virtual? Posteriormente, desde mediados del 2000 se comenzó a entender que en estas nuevas representaciones no se buscaban lugares sino más bien relaciones y flujos entre nodos que se transformaban permanentemente en escenarios de alteridad y multiplicidad.

Esto último permitió hablar no de educación virtual como un objeto, sino de virtualización de la educación entendida como proceso.

\section{Referencias bibliográficas}

Angel, F. 2008. La Educación Superior a Distancia en Colombia: un análisis del marco normativo. En C. Rama, M. Mena, \& A. Facundo, El Marco Regulatorio de la Educación Superior a Distancia en América Latina y el Caribe. Pp. 153-199. Bogotá D.C.: Ediciones Hispanoamericanas.

Angel, F. 2004. La educación virtual en Colombia. En C. Rama, La eduación virtual en América Latina situación y perspectivas. Pp. 165-217. Santiago de Chile: UNESCO.

Auge, M. 1995. Hacia una antropología de los mundos contemporáneos. Barcelona: Gedisa.

Bauman, Z. 2011. Collateral Damage: Social Inequalities in a Global Age. Cambridge: Polity Press.

Bauman, Z. 2007. Consuming Life. Cambridge: Polity Press.

Bauman, Z. 2005. Vida Líquida. Barcelona: Paidós.

Castells, M. 2010. End of Millennium. Oxford, U.K.: Blackwell Publishers.

Castells, M. 2002. La era de la información. Vol. I: La sociedad red. México Distrito Federal: Siglo XXI Editores.

Congreso de la República de Colombia. 1997. LEY 396 del 5 de agosto de 1997. «Por la cual se transforma la Unidad Universitaria del Sur de Bogotá, en Universidad Nacional Abierta y a Distancia - UNAD - y se dictan otras disposiciones». Bogotá D.C.: Diario Oficial No. 43.107, de 14 de agosto de 1997. 
Congreso de la República de Colombia. 1992. Ley 30 de diciembre 28 de 1992. por el cual se organiza el servicio público de la Educación Superior. Bogotá : Diario oficial de la Presidencia de la República.

Congreso de la República de Colombia. 1981. Ley 52 del 7 de julio de 1981. Bogotá D.C.: Diario Oficial de la república.

Díaz, R. 1995. Ritos mágicos, carabelas, computadoras personales: antropología y tecnología. Recuperado el 03 de Marzo de 2012 de, Nueva Antropología. Revista de Ciencias Sociales. Monográfico: Tecnología: de la ingeniería a la cultura. No. 47: disponible en: http://www.juridicas.unam.mx/publica/librev/rev/nuant/cont/47/cnt/ cnt2.pdf

Dominguez, D., Estaella, A., \& Gómez, B. 2007. Ethnography virtual. Recuperado el 15 de 04 de 2009, de Forum Qualitative Sozialforschung / Forum: Qualitative Social 8(3): disponible en: http://nbn-resolving.de/urn:nbn:de:0114-fqs0703E19.

Downes, S. 2012. The Rise of MOOCs. Recuperado el 25 de Enero de 2013, de Stephen Downes. stephen web's : disponible en: http://www.downes.ca/post/57911

Downes, S. 2008. El futuro del aprendizaje en línea: Diez años después. Recuperado el 26 de Septiembre de 2010, de Diego Leal : disponible en: http://www.diegoleal. org/social/blog/blogs/index.php/2009/06/17/stephen-downes-el-futuro-delaprendizaje?blog=2

Escobar, A. 2005. Bienvenidos a Cyberia. Notas para una Antropología de la cibercultura. Revista de Estudios Sociales. 22: 15-35.

Flórez, F. 2007. Michel F. et Jacques D.: ¿Antropólogos honoris causa? Notas para una genealogía de la «teoría social» posculturalista. Boletín de Antropología Universidad de Antioquia, 21(38): 381-390.

García, L. 2011. Perspectivas teóricas de la educación a distancia y virtual. Revista Española de Pedagogía. 249: 255-272.

González, B. 2006. Formación del tutor para la educación a distancia y los ambientes virtuales de aprendizaje en la universidad colombiana. 1974-2002. Bogotá D.C.: Universidad Javeriana.

Hine, C. 2008. Systematics as Cyberscience: Computers, Change, and Continuity in Science. Masachussets: MIT.

Hine, C. 2007. News infrastructures for Knowledge production: Understanding E-Sciencie. Londres: Idea Gropup Inc.

Hine, C. 2005. Virtual methods. Issues in social research on the Internet. Oxford: Berg. 
Entre flujos y fronteras: la educación superior mediada tecnológicamente vista a través de una perspectiva etnográfica . Artículo producto de la investigación.

Hine, C. 2004. Etnografía virtual. Barcelona: UOC.

Hirsh, R. 2011. Historians of Technology in the Real World. Reflections on the Pursuit of Policy-Oriented History. Recuperado el 03 de Marzo de 2012, de Revista Tecnología y Cultura. Revista de la Sociedad de historia de la tecnología. Universidad johns Hopkins: disponible en: http://muse.jhu.edu/journals/technology_and_culture/toc/tech.52.1.

Lopera, C. 2002. La Educacion Superior en Colombia. Digital Observatory for Higher Education in Latin America and the Caribbean IESALC. Bogotá: Unesco .

Maldonado, L. \& Leal, L. 2009. Análisis de interacciones en foros y chat: consolidación de grupo y liderazgo cmunicativo en un curso de lógica matematica, en Revista Iberoamericana de Educación a Distancia, 12(2): 189-210.

Maldonado, L., Leal, L., Perez, J., Montenegro, M., \& Castañeda, J. 2006. Sincronía y aprendizajes previos: Efectos sobre el aprendizaje y la consolidación de grupos en aulas virtuales. Revista de Investigaciones UNAD, 5:265-270.

Maldonado, L., \& Maldonado, P. 2000. Nuevas tecnologías aplicadas a la educación. Estado del arte de la investigación. 1990-1999. Bogotá: ICFES.

Martínez, A. 2004. De la escuela expansiva a la escuela competitiva: Dos modos de modernización en América Latina. (Primera ed.). Bogotá: Anthropos. Convenio Andrés Bello.

Mayans, J. 2002. Genero chat o como la etnografia puso un pie en el ciberespacio. Barcelona: Gedisa.

Mayans, J. 2002. Nuevas Tecnologías, Viejas Etnografías. Objeto y método de la antropología del ciberespacio. (C. d. Cibersociedad, Ed.) Recuperado el 08 de 03 de 2012, de Revista Quaderns de l'ICA, 17-18: disponible en: http://www.cibersociedad. net/archivo/articulo.php?art=23

Ministerio de Gobierno. 1987. Decreto No. 1782 del 17 de septiembre de 1987. Bogotá D.E.: Diario Oficial.

Moon, S. 2011. Accepting the Baton. Edittor's Note. Tecnología y Cultura, 1-5.

Observatorio para la CiberSociedad (OCS). 2013. NODOS I+D+A del OCS. (J. Mayans, Ed.) Recuperado el 15 de Febrero de 2013, de Observatorio para la CiberSociedad (OCS): disponible en: http://www.cibersociedad.net//nodos/index.php

Pinilla, P. 2000. Basicamente. Revista de la Facultad de Ciencias Básicas. Recuperado el 17 de agosto de 2009, de Universidad Santo Tomás: disponible en: http://basicamente. usta.edu.co/anteriores/numero1/Articulos/Virtualidad/Virtualidad.htm>

Pita , B. 2006. La universidad Santo Tomás y su contribución en el campo de la Educación a Distancia en Colombia. Revista Iberoamericana de Educación a Distancia, 93-125. 
Presidencia de la República de Colombia. 1997. Decreto 3011 del 19 de diciembre de 1997. por el cual se establecen normas para el ofrecimiento de la educación de adultos y se dictan otras disposiciones. Bogotá D.C.: Presidencia de la República.

Presidencia de la República. 1983. Decreto 1820 del 28 de junio de 1983. Por el cual se reglamente la Educación Abierta y a Distancia. Bogotá: Diario Oficial .

Presidencia de la República de Colombia. 1982. Decreto número 2412 de agosto 19 de 1982. por el cual se reglamenta, dirige e inspecciona la Educación Abierta y a Distancia y se crea el consejo de Educación Abierta y a Distancia. Bogotá D.C.: Diario Oficial .

Presidencia de la República. 1980. Decreto Ley 80 de 1980. Por el cual se organiza el sistema de educación post-secundaria. Bogotá D.C.: Diario Oficial de la Nación.

Rama, C. 2006. La despresencialización de la educación superior en América Latina: ¿Tema de calidad, de cobertura, de internacionalización o de financiamiento? Recuperado el 24 de 10 de 2010, de disponible en: http://www.claudiorama.name/sites/default/ files $/ 2-4 \% 20$ La\%20 despresencializacion $\% 20$ de $\% 20$ la $\% 20$ educacion $\% 20$ superior $\% 20$ en\%20Am\%C3\%Agrica\%20Latina.\%20tema\%20de\%20calidad,\%20cobertura,\%2ointernacionalizacion\%200\%20de\%20financiamiento.pdf

Restrepo, E. 2006. Teoría social, antropología y desarrollo: a propósito de narrativas y gráficas de Arturo Escobar. Boletín de Antropología Universidad de Antioquia., 20(37): 307-326.

Restrepo, E. 2008. Cuestiones de método: «eventualización»y problematización en Foucault. Tabula Rasa, 8: 111-132.

Rojas, J. 2013. Prácticas. Civilizar, Revista de Ciencias sociales de la Universidad Sergio Arboleda.

Rojas, J., \& Bernal, C. 2008. Etnografía aplicada en ambientes digitales: una experiencia metodológica en dos proyectos de doctorado en el contexto de la Educación Superior en Colombia. Hallazgos. Revista de al Unidad de Investigación de la Universidad Santo Tomas. (9): 167-189.

Rojas, J. 2003. La educación a distancia en Colombia, una historia que vale la pena contar. En J. Rojas m, \& W. Ortiz R, Ideas para integrarse a la educación a distancia. Pp. 105-116. Bogotá D.C.: UNAD.

Rojas, J. 2013. Educación virtual: del discurso teórico a las prácticas pedagógicas en la educación superior colombiana. UNED. Madrid: UNED. Recuperado el 02 de 2015, disponible en: http://e-spacio.uned.es/fez/eserv.php?pid=tesisuned:EducacionJerojas\&dsID=Documento.pdf

Rojas, J., \& Salazar, J. 2012. Cibercultura: una forma contemporánea de comunicación multimedia. (M. Palacios, Ed.) Análisis. Revista de investigaciones del Departamento de Humanidades. Universidad Santo Tomas, (en imprenta). 
Entre flujos y fronteras: la educación superior mediada tecnológicamente vista a través de una perspectiva etnográfica . Artículo producto de la investigación.

Ronderos, P., \& Valderrama, A. 2003. El futuro de la tecnología: una aperoximación desde la historiografía. Recuperado el 03 de Marzo de 2012, de Revista Iberoamericana de Ciencia Tecnología Sociedad e Información: disponible en: http://www.oei.es/ revistactsi/numero5/articulo5.htm

Rozo, C. 2008. El horizonte de la virtualidad. Tras la creación de lo posible. En C. Rozo, Contextos y pretextos sobre la pedagogía. Pp. 21-39. Bogotá: Universidad Pedagógica Nacional.

Rueda, R. (2008). Cibercultura: metaforas practicas sociales y colectivos en red. Nomadas, 8-20.

Sarmiento, L. 2007. Modelo colombiano de Educación Abierta y a Distancia SED. Revista Colombiana de Historia de la Educación, 73-100.

Staudenmaier, J. 2002. Rationality, Agency, Contingency: Recent Trends in the History of Technology. Recuperado el 04 de Marzo de 2012, de Reviews in American History, 30 (1): 168-181: disponible en: http://www.jstor.org/stable/30031729

Tamayo, A. 2006. El movimiento pedagógico en Colombia: un encuentro de los maestros con la pedagogía. Revista HISTEDBR On-line Campinas, 102-113. Obtenido de Revista HISTEDBR On-line Campinas: disponible en: http://www.histedbr.fae.unicamp.br/ artog_24.pdf

Turkle, S. 2011. Alone Together: Why we expect more from technology and less from each other. Nueva York, EEUU: Basic Books.

Turkle, S. 2009. Simulation and its Discontens. Cambridge: MIT, Press.

Turkle, S. 2007. Aunthenticity in the age of digital companions. Interaction studies, 8(3): 501-517.

Turkle, S., \& Papert, S. 1991. Epistemological pluralism and the revaluation of the concrete. En S. Papert, Constructinism, Research, report and essays, 1985-1990. Pp. 161189. Massachusetts: M.I.T.

Turkle, S., Taggart, W., \& Kidd, C. 2006. A sociable Robot to Encourage Social Interaction among the Elderly. En I. C. Automation (Ed.), A sociable Robot to Encourage Social Interaction among the Elderly. Pp. 3972-3976. Orlando- Florida: IEEE.

UNESCO. 1996. La Educación encierra un tesoro Informe a la UNESCO de la Comisión Internacional sobre la educación para el siglo XXI, presidida por Jack Delors. Madrid: Santillana.

UNESCO. 1989. 4la Reunión de la Conferencia Internacional de la Educación. Dirigida a los miembros de los ministerios de Educación, relativa a la «Diversificación de la enseñanza Postsecundaria ante la situación del empleo». Ginebra: Unesco. 
UNESCO. 1949. Primera confererencia mundial sobre la Eduación de Adultos. Elsinor: Unesco.

UPN. 2006. Proyecto Político Pedagógico UPN. Bogotá D.C.: UPN.

USTA. Vicerrectoría Académica. 2009. Modelo Educativo Pedagógico. Bogotá: USTA.

USTA. 2004. Proyecto Educativo Institucional -PEI-. Bogotá D.C.: USTA.

Velasco, H., \& Díaz de Rada, A. 1997. La lógica de la investigación etnográfica: un modelo de trabajo para etnógrafos de escuela. Madrid: Editorial Trotta. 